\title{
Crystalline Orientation Effects on Ballistic Hole Current in Ultrathin DG SOI MOSFETs
}

\author{
H. Minari and N. Mori \\ Graduate School of Engineering, Osaka University \\ 2-1 Yamada-oka, Suita City, Osaka 565-0871, Japan \\ \{minari|mori\}@e3.eei.eng.osaka-u.ac.jp
}

\begin{abstract}
Atomistic hole transport simulation based on a nonequilibrium Green's function method and a tight-binding approximation has been performed for two types of ultrathin doublegate silicon-on-insulator MOSFETs; (i) $\langle 100\rangle$-device on a $\{100\}$ substrate where the current flows along the $\langle 100\rangle$ direction and (ii) $\langle 110\rangle$-device on a $\{110\}$ substrate where the current flow direction is the $\langle 110\rangle$ direction. Simulation results show that the difference in crystalline orientation of the devices greatly affects ballistic hole current due to a strong confinement-induced mixing of heavy- and light-hole states.
\end{abstract}

\section{Introduction}

A channel length of Si MOSFETs continues to shrink rapidly down to a sub-10-nm regime. In such an ultra-small device, quantum mechanical effects, such as direct source-to-drain tunneling and subband quantization along the gate-confinement direction, significantly affect the transport characteristics. The nonequilibrium Green's function (NEGF) method $[1,2]$ allows us to calculate quantum transport characteristics in ultra-small MOSFETs. By incorporating a tight-binding approximation (TBA) into the NEGF formalism one can achieve quantum-mechanical computations with atomic resolution [3]. We have reported on atomistic modeling for one-dimensional Si nanostructures within the framework of the NEGF formalism and an empirical TBA $[4,5]$. In the present study, we have performed two-dimensional simulation of hole transport in double-gate (DG) silicon-on-insulator (SOI) MOSFETs. We especially focus our study on crystalline orientation effects on ballistic hole current. The main differences from a similar work [6] are (i) the tight-binding band-structure is taken into account not only for the transport direction but also for the transverse direction, (ii) $p$-type devices are considered, and (iii) crystalline orientation effects are examined. Since the subband dispersion in an ultrathin body SOI structure is quite nonparabolic and anisotropic due to the strong confinement-induced mixing of heavy- and light-hole states [7], it is essential to include the in-plane full-band structure into calculation.

\section{Simulation Method}

We consider $p$-type DG SOI MOSFETs with a gate-length of $8 \mathrm{~nm}$, a body-thickness of $3 \mathrm{~nm}$, and $\mathrm{SiO}_{2}$ thicknesses of $0.5 \mathrm{~nm}$ [8] (see Fig. 1). The gates are assumed to consist 


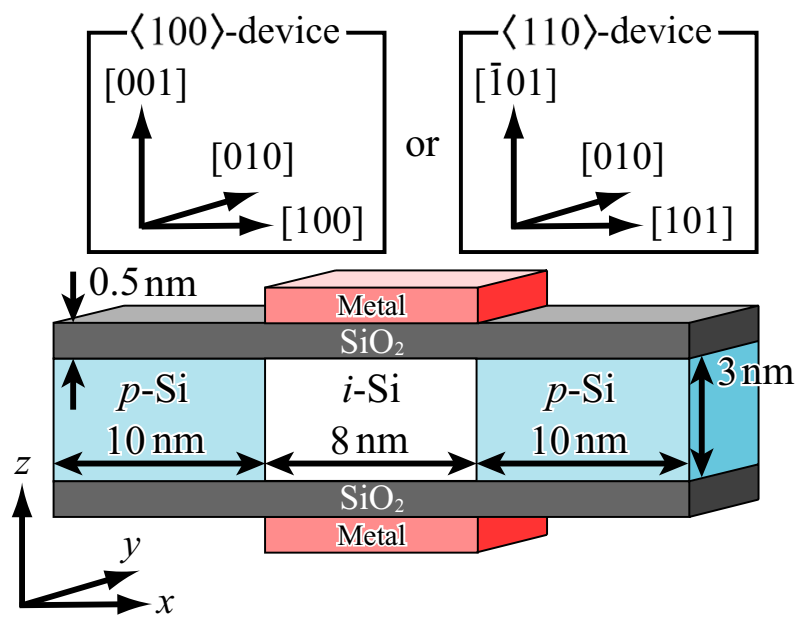

Figure 1: Schematic diagram of the DG SOI MOSFET structure together with the coordinate system. We consider two types of devices; (i) $\langle 100\rangle$-device on a $\{100\}$ substrate where the current flows along the $\langle 100\rangle$ direction and (ii) $\langle 110\rangle$-device on a $\{110\}$ substrate where the current flow direction is the $\langle 110\rangle$ direction.

of the mid-gap metal. The doping concentration in the $p$-regions, each of which is $10 \mathrm{~nm}$ long, is $1 \times 10^{20} \mathrm{~cm}^{-3}$. To study crystalline orientation effects on the ballistic hole current, we simulated two types of devices; (i) $\langle 100\rangle$-device on a $\{100\}$ substrate where the current flows along the $\langle 100\rangle$ direction and (ii) $\langle 110\rangle$-device on a $\{110\}$ substrate where the current flow direction is the $\langle 110\rangle$ direction (see Fig. 1). We took into account of the valence-band structure within the $\mathrm{sp}^{3} \mathrm{~s}^{*}$ empirical TBA [9] and calculated ballistic hole current using the NEGF method.

\section{Results and Discussion}

For an ultrathin Si slab, the degeneracy of heavy- and light-holes is lifted due to subband quantization; the light-holes have the higher subband energy and the heavy-holes have the lower subband energy. On the other hand, the light-holes have the larger transverse mass and the heavy-holes have the smaller transverse mass. This results in the confinement-induced mixing of heavy- and light-hole states [7], which makes the hole subband structure quite nonparabolic and anisotropic. Figs. 2(a) and 2(b) show the calculated subband dispersion for $\langle 100\rangle$ - and $\langle 110\rangle$-devices, respectively. The nonparabolicity of the subband is tremendous compared to a simple parabolic dispersion shown in Fig. 2(c), where we assume independent heavy- and light-holes with $m_{\mathrm{hh}}=0.53 m_{0}$ and $m_{\mathrm{lh}}=0.155 m_{0}$, respectively [10]. We also see that some excited subbands have a dispersion which decreases with increasing $k$ when $k$ is small. This kind of carriers cannot be accurately handled within a simple effective mass approximation. It is, therefore, essential to include the in-plane full-band structure into calculation for simulation of ultrathin SOI MOSFETs. 

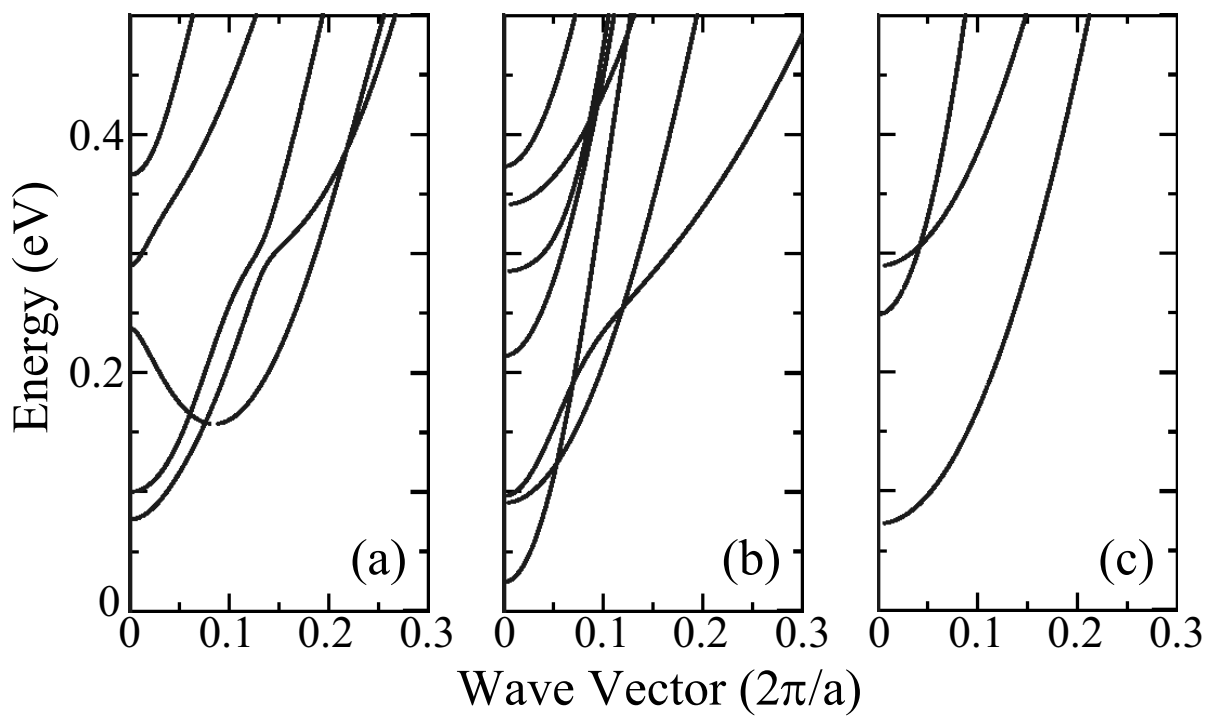

Figure 2: Subband dispersion of a $3 \mathrm{~nm}$-thick Si quantum-well for (a) $\langle 100\rangle$-device and (b) $\langle 110\rangle$-device. (c) A simple parabolic dispersion for independent heavy-and light-holes.

Fig. 3 shows calculated results of potential profiles, hole density spectra, and current density spectra at gate voltage $V_{\mathrm{GS}}=-0.8 \mathrm{~V}$, drain voltage $V_{\mathrm{DS}}=-50 \mathrm{mV}$ and $T=$ $300 \mathrm{~K}$ for (a) $\langle 100\rangle$ - and (b) $\langle 110\rangle$-devices. The energy zero is chosen to be the Fermi level in the source contact. The potential profiles are obtained through a self-consistent solution of Poisson and TBA-NEGF equations. For TBA-NEGF, we treated the $\mathrm{Si} / \mathrm{SiO}$ interfaces with the $\mathrm{H}$ termination model [11] for eliminating the nonphysical surface states in the energy region of interest. We find significant difference between the hole density spectrum of the $\langle 100\rangle$-device and that of the $\langle 110\rangle$-device. This difference is originated not only in the anisotropic dispersion in bulk Si but also in the confinementinduced mixing of heavy- and light-hole states in ultrathin SOI structures. We also find that the current density of the $\langle 110\rangle$-device is 29 percent larger than that of the $\langle 100\rangle$ device. This is because the effective mass in the $\langle 100\rangle$-device is much heavier than that of the $\langle 110\rangle$-device at lower energy region (see Fig. 2).

\section{Conclusions}

We performed atomistic transport simulation based on the NEGF method combined with the $\mathrm{sp}^{3} \mathrm{~s}^{*}$ empirical TBA for two types of DG SOI MOSFETs that have different crystalline orientation for the current flow and confining directions. We find that the difference in the crystalline orientation greatly affects the ballistic hole current due to the strong confinement-induced mixing of heavy- and light-hole states in an ultrathin body SOI structure. 


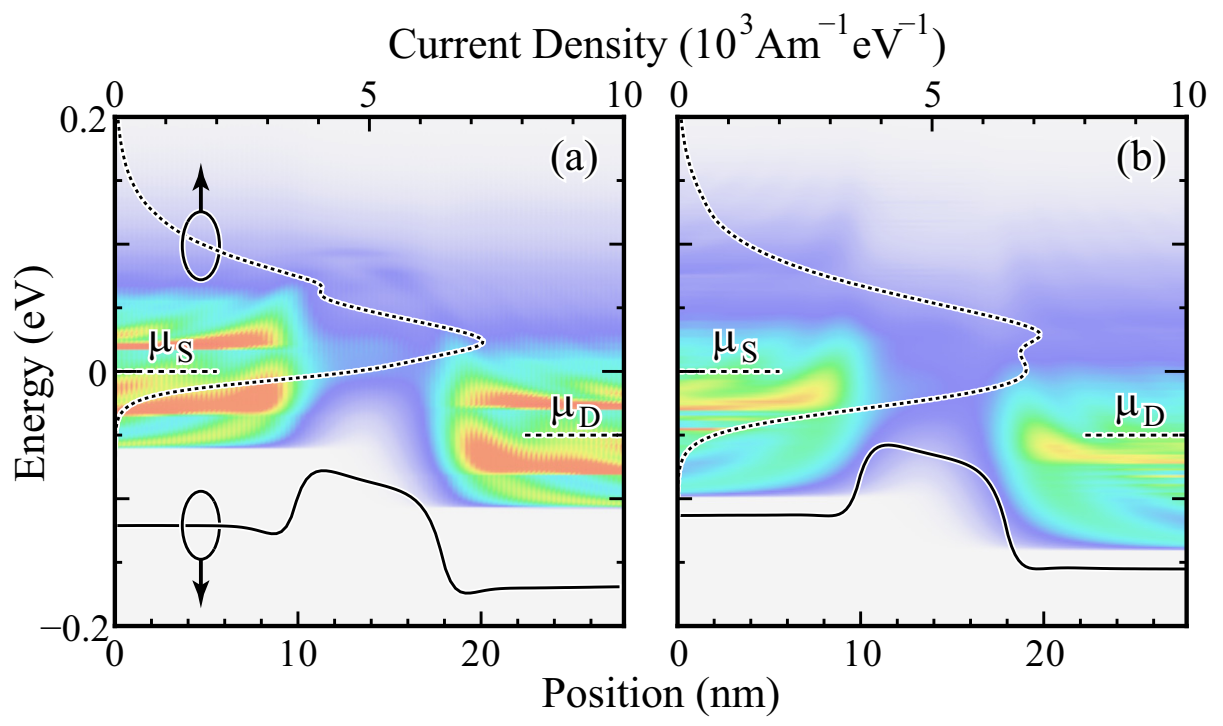

Figure 3: Calculated hole density spectra (colored plot) for (a) $\langle 100\rangle$-device and (b) $\langle 110\rangle$-device. Solid lines show the potential profiles, dotted lines the current density spectra and dashed lines Fermi levels in the source $\left(\mu_{\mathrm{S}}\right)$ and drain $\left(\mu_{\mathrm{D}}\right)$ contacts.

\section{Acknowledgments}

The authors gratefully acknowledge useful and insightful discussion with Hideki Oka, Kiyoshi Ishikawa, Hiroshi Takeda, and Shinya Yamakawa. This work was financially supported by the Semiconductor Technology Academic Research Center (STARC).

\section{References}

[1] S. Datta, Electronic Transport in Mesoscopic Systems, (Cambridge University Press, Cambridge, UK, 1995).

[2] M. Lundstrom and J. Guo, Nanoscale Transistors: Device Physics, Modeling, and Simulation, (Springer, New York, 2006).

[3] A. Pecchia and A. Di Carlo, Rep. Prog. Phys., 64, 1497 (2004).

[4] H. Minari and N. Mori, Jpn. J. Appl. Phys., 46, 2076 (2007).

[5] H. Minari and N. Mori, J. Comput. Electron, 6, 223 (2007).

[6] T. Xia, L. F. Register, and S. K. Banerjee, IEEE Trans. Electr. Dev., 50, 1511 (2003).

[7] C. Weisbuch, Fundamental Properties of III-V Semiconductor Two-Dimensional Quantized Structures: The Basis for Optical and Electronic Device Applications, R. Dingle, ed., Semicond. Semimet. 24, 1 (1987).

[8] H. Tsuchiya, K. Fujii, T. Mori, and T. Miyoshi, IEEE Trans. Electr. Dev., 53, 2965 (2006).

[9] P. Vogl, H. P. Hjalmarson, and J. D. Dow, J. Phys. Chem. Solids, 44, 365 (1983).

[10] C. Jacoboni and L. Reggiani, Rev. Mod. Phys., 55, 645 (1983).

[11] S. Lee, F. Oyafuso, P. V. Allmen, and G. Klimeck, Phys. Rev. B, 69, 045316 (2004). 